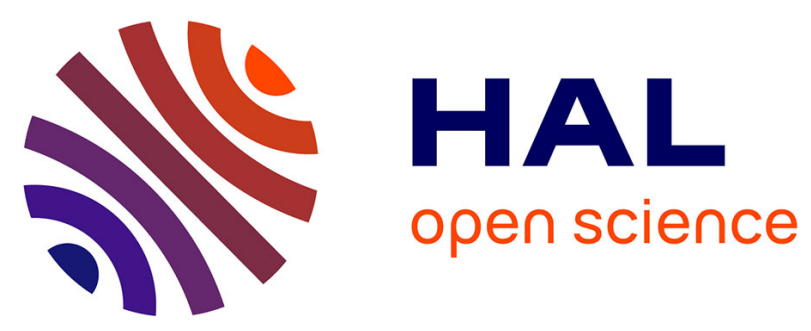

\title{
Influence of composition on the morphology of polyurethane/acrylic latex particles and adhesive films
} Elise Degrandi-Contraires, Ravindra Udagama, Timothy Mckenna, Elodie Bourgeat-Lami, Christopher J.G. Plummer, Costantino Creton

\section{- To cite this version:}

Elise Degrandi-Contraires, Ravindra Udagama, Timothy Mckenna, Elodie Bourgeat-Lami, Christopher J.G. Plummer, et al.. Influence of composition on the morphology of polyurethane/acrylic latex particles and adhesive films. International Journal of Adhesion and Adhesives, 2014, 50, pp.176 - 182. 10.1016/j.ijadhadh.2014.01.025 . hal-01516058

\section{HAL Id: hal-01516058 https://hal.sorbonne-universite.fr/hal-01516058}

Submitted on 28 Apr 2017

HAL is a multi-disciplinary open access archive for the deposit and dissemination of scientific research documents, whether they are published or not. The documents may come from teaching and research institutions in France or abroad, or from public or private research centers.
L'archive ouverte pluridisciplinaire HAL, est destinée au dépôt et à la diffusion de documents scientifiques de niveau recherche, publiés ou non, émanant des établissements d'enseignement et de recherche français ou étrangers, des laboratoires publics ou privés. 


\title{
Influence of composition on the morphology of polyurethane/acrylic latex particles and adhesive films
}

\author{
Elise Degrandi-Contraires ${ }^{\mathrm{a}, 1}$, Ravindra Udagama ${ }^{\mathrm{b}}$, Timothy McKenna ${ }^{\mathrm{b}}$, \\ Elodie Bourgeat-Lami ${ }^{\mathrm{b}}$, Christopher J.G. Plummer ${ }^{\mathrm{c}}$, Costantino Creton ${ }^{\mathrm{a}, \mathrm{*}}$ \\ a Laboratoire de Sciences et Ingénierie de la Matière Molle, CNRS, ESPCI-ParisTech, UPMC, 10, rue Vauquelin, F-75231 Paris cedex 05, France \\ ${ }^{\mathrm{b}}$ Université de Lyon, Université Lyon 1, CPE Lyon, CNRS, UMR 5265, Laboratoire de Chimie Catalyse Polymères et Procédés (C2P2), LCPP Team, Bat 308F, \\ 43 Bd du 11 novembre 1918, F-69616 Villeurbanne, France \\ ${ }^{\mathrm{c}}$ Laboratory of Polymer and Composite Technology (LTC), Ecole Polytechnique Fédérale de Lausanne (EPFL), Station 12, Lausanne CH-1015, Switzerland
}

\section{A R T I C L E I N F O}

\section{Article history:}

Accepted 22 January 2014

Available online 31 January 2014

Keywords:

Hybrid PSA

Mechanical properties

Morphology

\begin{abstract}
A B S T R A C T
Polyurethane (PU)/acrylic hybrid particles with different PU contents were synthesized by miniemulsion polymerization and subsequently dried to give solid adhesive films. The morphologies of the particles and the morphologies and mechanical properties of the resulting films were investigated by Transmission electron microscopy combined with selective staining of the PU and by uniaxial tension tests. Morphological investigations showed a clear change in the particle morphology as the PU weight fraction increased. While at $5 \mathrm{wt} \%$ and $25 \mathrm{wt} \% \mathrm{PU}$ (with respect to total organic content) the particles were relatively homogeneous and mechanical properties of the films could be readily interpreted with molecular arguments, at $50 \mathrm{wt} \% \mathrm{PU}$ a core-shell structure was observed. This heterogeneous structure of the $50 \mathrm{wt} \%$ PU particles persisted in the films, resulting in a percolating network of the harder PU phase. The low deformability and strain at failure of the 50\% PU films suggest that, unlike the adhesives with lower PU content, the relatively weak interfaces between the original latex particles dominate the mechanical properties.
\end{abstract}

(c) 2014 Elsevier Ltd. All rights reserved.

\section{Introduction}

In coating applications, polyurethane (PU) improves properties of waterborne acrylic latexes such as resistance to solvent, film formation or toughness [1-3]. Given the attractive properties of PU-modified films, such systems have been widely studied since the end of the 1990s [4-11]. Miniemulsion polymerization is considered to be one of the most promising routes to the production of PU/acrylic hybrid particles because of the manner in which the particles are formed. Ideally it is possible to create droplets of acrylic monomer and PU precursors, and then polymerize each droplet to create a homogeneous dispersion of hybrid particles [3,5-7,10]. In addition, Li et al. [3] showed that the covalent grafting of the PU chains onto the acrylic backbone is an efficient method to reduce the macroscopic phase separation that can occur in solution-based processes between PU and acrylic because of their limited miscibility. Such work in coatings has been recently extended to waterborne acrylic adhesives where PU is added mainly to increase cohesion of the adhesive films [12-18]. All acrylic PSA are materials with glass transition temperatures $\left(T_{\mathrm{g}}\right)$ well

\footnotetext{
* Corresponding author

${ }^{1}$ Current address: Laboratoire de Tribologie et Dynamique des Systèmes (LTDS), CNRS UMR 5513, Ecole Centrale de Lyon, 36 avenue Guy de Collongue, F-69134 Ecully cedex, France.
}

below room temperature, and have very low entanglement densities. The addition of PU is beneficial not only because it improves properties such as solvent resistance, but may also increase the entanglement density of the polymer phase and thus the shear resistance. In addition it helps to increase creep resistance and therefore improves adhesion at high temperatures [19].

In these studies, to avoid macroscopic phase separation between PU and the acrylic polymer, the technique of covalent grafting was validated in a one-step polymerization process by Lopez and coworkers [12,18], as well as in a two-step process that included an initial step in which the PU chains were capped with hydroxyethylmethacrylate (HEMA), and a second step in which the resulting miniemulsions were polymerized [17].

In one of these studies, we reported the synthesis of model PU/ acrylic hybrid latexes by miniemulsion polymerization [17]. In a companion paper, we investigated the impact of different process parameters (PU content, quantity of chain extender, etc.) on the mechanical properties of the films obtained from these latexes [15]. The results led to the conclusion that the degree of grafting and the PU chain length (controlled by the ratio of the grafting agent HEMA to the chain extender Bisphenol A (BPA)) played an important role in the architecture of the insoluble fraction. More importantly for the present study, it was shown that the hypothesis of fully homogeneous particle morphology was insufficient to 
explain the mechanical test results satisfactorily. We felt therefore that investigations of the morphology of the hybrid particles were necessary to complete our analysis.

Transmission electron microscopy (TEM) was used in the present work to examine the particle and film morphologies in detail. Because particles and films are soft, with $T_{\mathrm{g}}$ much lower than ambient temperature, sample preparation and observation are not trivial and require strict protocols. Acrylic or PU phases can however be selectively stained to improve contrast; ruthenium tetraoxide or osmium tetraoxide preferentially stain the acrylic phase (enriched with polystyrene) [6,20,21], whereas phosphotungstic acid (PTA) can be used to stain the PU $[3,11,22]$. This last method was employed here. The mechanical properties of the adhesive films were then reconsidered in the light of the observed morphology of the PU/acrylic hybrid particles and the films.

\section{Experimental section}

\subsection{Synthesis}

All PU/acrylic hybrid latexes have been prepared by miniemulsion polymerization as reported elsewhere [17]. A base composition of butyl-acrylate (BA; $89.5 \mathrm{wt} \%)$, methyl-methacrylate (MMA; $9.5 \mathrm{wt} \%$ ) and acrylic acid (AA; $1 \mathrm{wt} \%$ ) was used for the acrylic copolymer. The polyurethane prepolymer (PU, Incorez 701) was provided by Industrial Copolymers Limited. Molecular analyses have demonstrated that this prepolymer is made up of polypropylene glycol (PPG) for the soft segments and isophorone diisocyanate (IPDI) for the hard ones. Its molar mass was checked by size exclusion chromatography and inversed titration of the isocyanate functions and was found to be $\sim 3000 \mathrm{~g} \mathrm{~mol}^{-1}$. Different PU weight fractions related to monomers were incorporated into the droplets during polymerization (5 wt\%, $25 \mathrm{wt} \%$ and $50 \mathrm{wt} \%)$.

The PU chains were NCO-terminated at both ends to react with alcohol functions of HEMA, which reacted also during the radical polymerization of the acrylic monomers playing the role of a grafting agent. A calculated amount of HEMA was added to have a grafting degree of the PU equivalent to $10 \mathrm{wt} \%$ of the NCO functions. The remaining free isocyanate functions were neutralized with BPA as a chain extender. The samples were synthesized in three stages: the PU was mixed in the acrylic phase and reacted first with the HEMA, then the remaining NCO moieties were neutralized by the alcohol functions of BPA (with a final ratio $\mathrm{NCO} / \mathrm{OH}$ of 1.82 ) and this organic phase was miniemulsified by sonication. Finally the free radical polymerization was performed by miniemulsion in a batch process. Table 1 shows the main characteristic of the samples synthesized in this work. More details on chemistry of these systems can be found in our previous paper [15].

Table 1

Main characteristics of the PU/acrylic latex samples synthesized in this work.

\begin{tabular}{rlllll}
\hline Sample (PU) & $\begin{array}{l}\text { PU } \\
\text { (wt\%) }\end{array}$ & NCO/OH & $\begin{array}{l}\text { Degree of } \\
\text { grafting (\%) }\end{array}$ & $\begin{array}{l}\text { Particle } \\
\text { diameter }(\mathrm{nm})^{\mathrm{b}}\end{array}$ & $\begin{array}{l}\text { Gel } \\
\text { content (\%) }\end{array}$ \\
\hline 5 & 5 & 1.82 & 10 & 94 & $/^{\mathrm{c}}$ \\
25 & 25 & 1.82 & 10 & 121 & 37.4 \\
50 & 50 & 1.82 & 10 & 132 & 73.8 \\
\hline
\end{tabular}

a Theoretical value based on the hypothesis of complete reaction between PU and HEMA.

${ }^{\mathrm{b}}$ Measured by DLS (ZETASIZER 1000 HSA).

${ }^{\mathrm{c}} \mathrm{Gel}$ content too low to be measured with the technique used in this paper.

\subsection{Characterization}

Average droplet $\left(D_{\mathrm{d}}\right)$ and particle sizes $\left(D_{\mathrm{p}}\right)$ were measured by photon correlation spectroscopy at $90^{\circ}$ (ZETASIZER $1000 \mathrm{HSA}$ ). Average sizes reported here are the averages of at least 5 measurements per sample. Glass transition temperature was determined by differential scanning calorimetry (DSC, TA Instrument DSC 2920).

Gel content and swelling ratio were measured by static measurements. The latex was dried in a mold to obtain a self-standing film. A small piece of dried latex $(\sim 0.1 \mathrm{~g})$ was cut and weighed $\left(W_{1}\right)$. Then, it was immersed in THF for a week. After a week, the sample completely swollen by the solvent was taken out and weighed again. It was then dried at $60{ }^{\circ} \mathrm{C}$ for $30 \mathrm{~min}$ to extract all the solvent and the dried part was weighed $\left(W_{3}\right)$. The gel content was given by

$\%$ gel $=\frac{W_{3}}{W_{1}} \times 100$

\subsection{Imaging techniques}

For TEM imaging, particles were previously stained by phosphotungstic acid (PTA). $1.5 \mathrm{wt} \%$ of PTA was added to a solution of 1:80 diluted latex. The solution of diluted latex and PTA was sonicated for $30 \mathrm{~min}$ at maximum power. A small droplet of this solution was spin-coated onto a carbon covered 200 mesh copper grid at $1500 \mathrm{rpm}$ for $3 \mathrm{~min}$. To prepare films for TEM, latexes were cast in silicone molds and placed in an oven at $40{ }^{\circ} \mathrm{C}$ for $4 \mathrm{~h}$ until completely dried. For staining, small pieces cut from the films (several hundred $\mu \mathrm{m}$ in thickness) were placed in a solution containing $1.5 \mathrm{wt} \%$ of PTA. These were then sectioned with a cryo-ultramicrotome (Reichert-Jung Ultracut E/Diatome diamond knife) at approximately $-90^{\circ} \mathrm{C}$. The sections, of approximately $100 \mathrm{~nm}$ in thickness, were picked up on carbon covered 400 mesh copper grids for observation. A TEM Philips CM20 equipped with a $\mathrm{LaB}_{6}$ filament operating at $200 \mathrm{kV}$ was used throughout. All images were obtained at room temperature. For each sample, several images of several particles deposited on the carbon grid were taken. For the films, several images were taken at different places on samples of average size of $5 \mathrm{~mm}^{2}$. This validates the reproducibility of the results.

\subsection{Mechanical characterization}

Self-standing films dried in silicone molds were used for the tensile experiments. To control the thickness of these films, a calculated volume of latex was deposited depending on the solid content and the desired thickness. The molds were covered with a bell jar to control the rate of humidity and to protect them from dust. After a week, molds were placed for $5 \mathrm{~min}$ at $110{ }^{\circ} \mathrm{C}$ and the samples were then taken out, placed between low-release silicone papers and cut to the desired dimensions with a die-cutter.

A standard tensile INSTRON machine (model 5565) equipped with a video-extensometer (model SVE) was used. The machine used a $10 \mathrm{~N}$ load cell with a resolution of $\pm 1 \mathrm{mN}$. Rectangular strips were cut within the self-standing films. All of them were $5 \mathrm{~mm}$ wide $\left(w_{0}\right)$ with an average thickness $\left(e_{0}\right)$ of the order of $500 \mu \mathrm{m}$. Individual values were controlled for each sample. The initial velocity of the crosshead was chosen to obtain an initial strain rate of $0.1-1 \mathrm{~s}^{-1}$. All tests were performed at room temperature.

\section{Results and discussion}

The TEM images obtained for latex particles containing three different PU weight fractions are shown in Fig. 1. The darker areas 

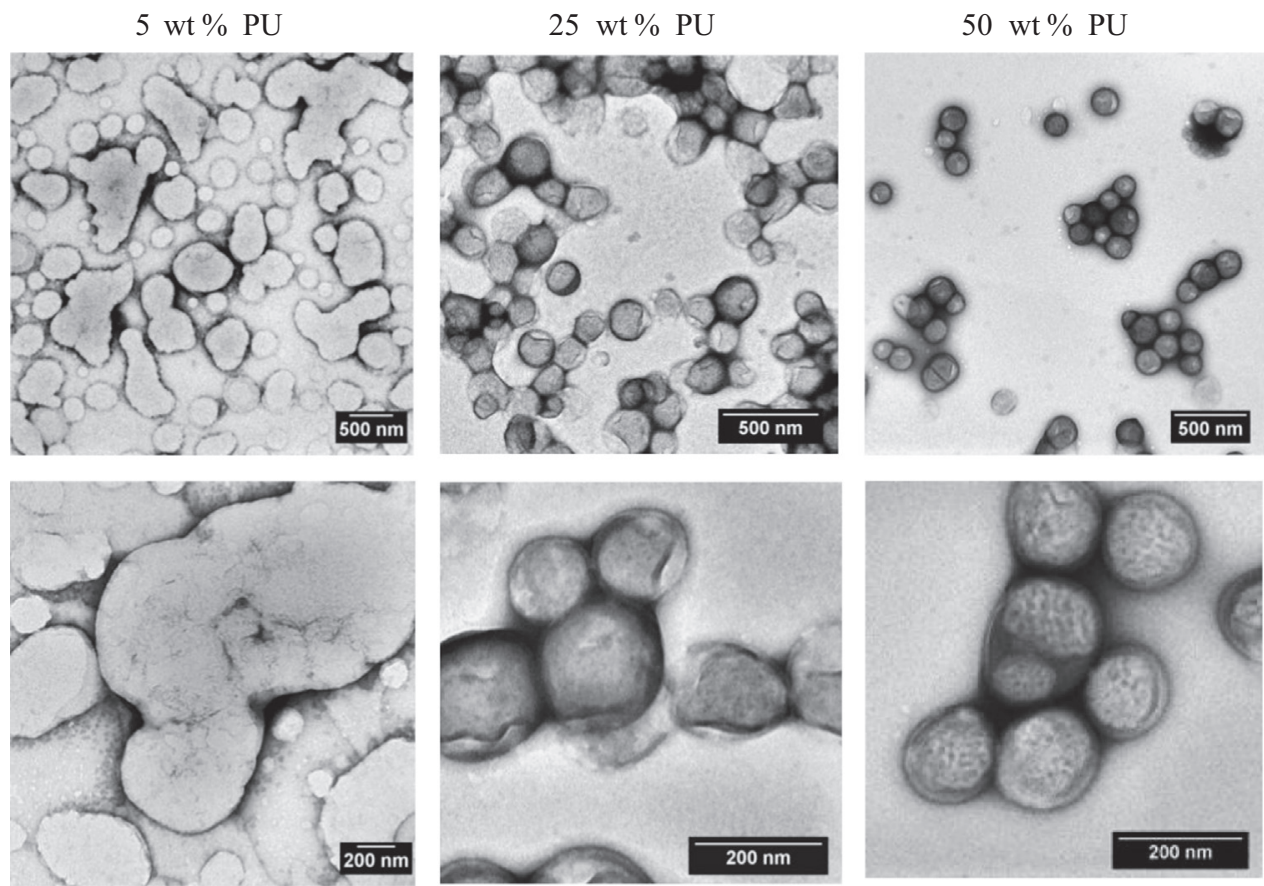

Fig. 1. TEM images of latex particles stained with PTA for three different PU weight fractions (5 wt\%: left, 25 wt $\%$ : center, and 50 wt $\%$ : right).

correspond to regions of relatively strong scattering and it is known that PTA (which has a relatively high effective scattering cross-section owing to the high atomic number of tungsten) preferentially stains the PU domains [23]. This results in PU domains appearing darker than the acrylic domains [11,22]. PTA also accumulates at the particle surfaces during drying, leading to negative staining of the particles and particle clusters themselves.

Clusters of particles were observed for each of the different PU weight fractions. For the lowest PU weight fraction ( $5 \mathrm{wt} \%$ ), the particles underwent considerable coalescence within the clusters and could not be clearly distinguished. For the higher PU fractions (25 wt\% and $50 \mathrm{wt} \%$ ), however, individual particles were clearly observed. For $25 \mathrm{wt} \% \mathrm{PU}$, the particles appeared to be nevertheless larger than those shown in Table 1. This is an indication that the particles are very soft and hence flatten on the carbon grid during deposition. The glass transition temperature of the materials is $-39.1 \pm 3.0^{\circ} \mathrm{C}$, regardless of the PU content. This value confirms that the samples are in the range of very soft materials at room temperature and is consistent with particles flattening. Only one $T_{\mathrm{g}}$ is measured for each sample, which in principle suggests that there is no large scale phase separation. However in this case $T_{\mathrm{g}} \mathrm{S}$ of both polymers are very close $\left(-48{ }^{\circ} \mathrm{C}\right.$ for the PU) with a wide distribution for pure acrylic's $T_{\mathrm{g}}$. It is then probable that a separation between two $T_{\mathrm{g}} \mathrm{s}$ would be masked by the vicinity of $T_{\mathrm{g}}$ of each polymer.

Fig. 1 shows that the final particle morphology is strongly dependent on the quantity of PU incorporated in the formulation. A schematic representation of the evolution of the morphology is proposed in Scheme 1. When $5 \mathrm{wt} \% \mathrm{PU}$ is added, the particles appear relatively homogeneous. At $25 \mathrm{wt} \% \mathrm{PU}$, large homogeneous regions rich in acrylic polymer appear, with distinct domains of PU in the outer regions that only cover a portion of the acrylic surface. Finally, at $50 \mathrm{wt} \% \mathrm{PU}$, a core-shell structure appears and one can distinguish dark spots in the core of the particles. The dark domains inside the core of these particles are also assumed to be PU rich, indicating a heterogeneous particle structure. In the case of the high PU-content particles, the observation that the particles exhibit a core-shell structure with a PU shell and an acrylic core agrees with previous studies [9-11,21,22,24]. However, we have found no other reports of isolated PU domains forming inside the particles as well. One possible explanation for this structure is that the method used to incorporate the PU by grafting onto the acrylic chains is not efficient enough to fully graft large amounts of PU to the acrylic polymer, so that heterogeneous particles with acrylicrich regions and PU-rich regions are detected.

Since every PU chain should be in principle grafted to the acrylic backbone one may argue that the observed shell may be PMMA that is more hydrophilic and can accumulate more easily at the particle borders. Nevertheless, the PMMA proportion in the acrylic monomer composition is independent of the PU content, so if the shells were PMMA their morphology should then be independent of the quantity of PU in the formulation which is not the case. Moreover, even though some PU chains are indeed grafted from both ends to the acrylic chains with HEMA, some are probably attached by one end only, and others may not be connected to the acrylic backbone at all [15]. The observed coreshell morphology indicates that at least part of the PU chains are dangling or free chains that can migrate to the surface of the particles during synthesis.

Self-standing dried latex films were observed by TEM and images are shown in Fig. 2. For particles with low quantities of PU, a homogeneous film with no apparent memory of the original particle structure is obtained. This confirms the incorporation of the PU at the molecular level and a uniform film formation. The film with 25 wt\% of PU is less homogeneous. A dark gray matrix is visible, in which white and black distinct domains are randomly distributed.

Since the film has been stained, the PU-rich domains are assumed to correspond to the black almost circular domains. The interpretation of the white domains is less clear, but they may correspond to damage due to sample preparation. Thus, the film remains relatively homogeneous with a random distribution of PU domains. For the highest PU weight fraction (50 wt\%), the core-shell structure of the particles is clearly maintained during film formation and can be observed in the TEM images. Also, for $50 \mathrm{wt} \% \mathrm{PU}$, the domain diameter estimated by fitting with Image $(131 \pm 40 \mathrm{~nm})$ is consistent with the particle diameter measured by DLS for $50 \mathrm{wt} \%$. 


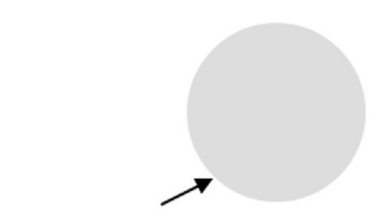

Homogeneous PU/acrylic particle

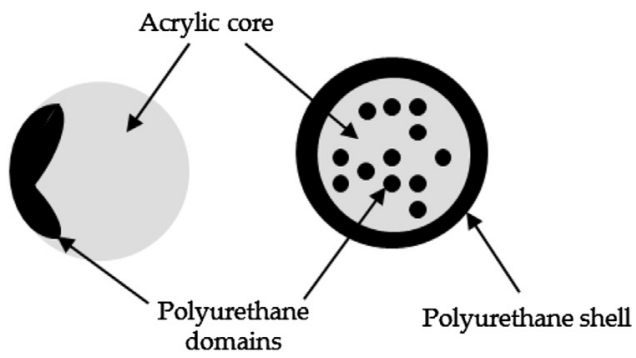

Increasing PU weight fraction

Scheme 1. Schematic representation of the particles for increasing PU weight fractions.

$5 \mathrm{wt} \% \mathrm{PU}$

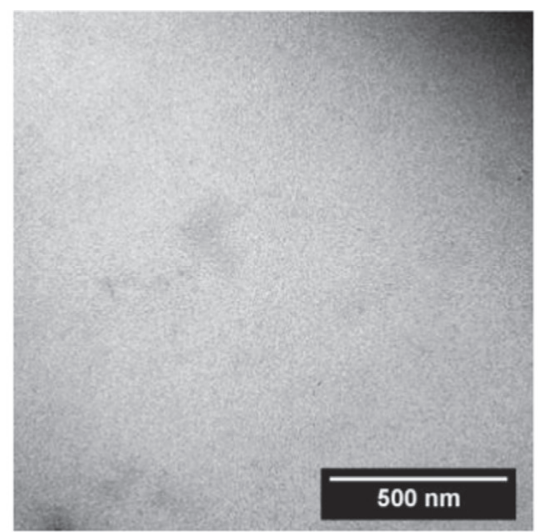

$25 \mathrm{wt} \% \mathrm{PU}$

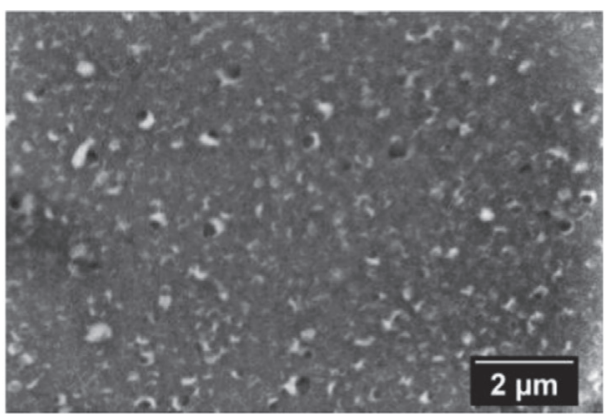

$50 \mathrm{wt} \%$ PU
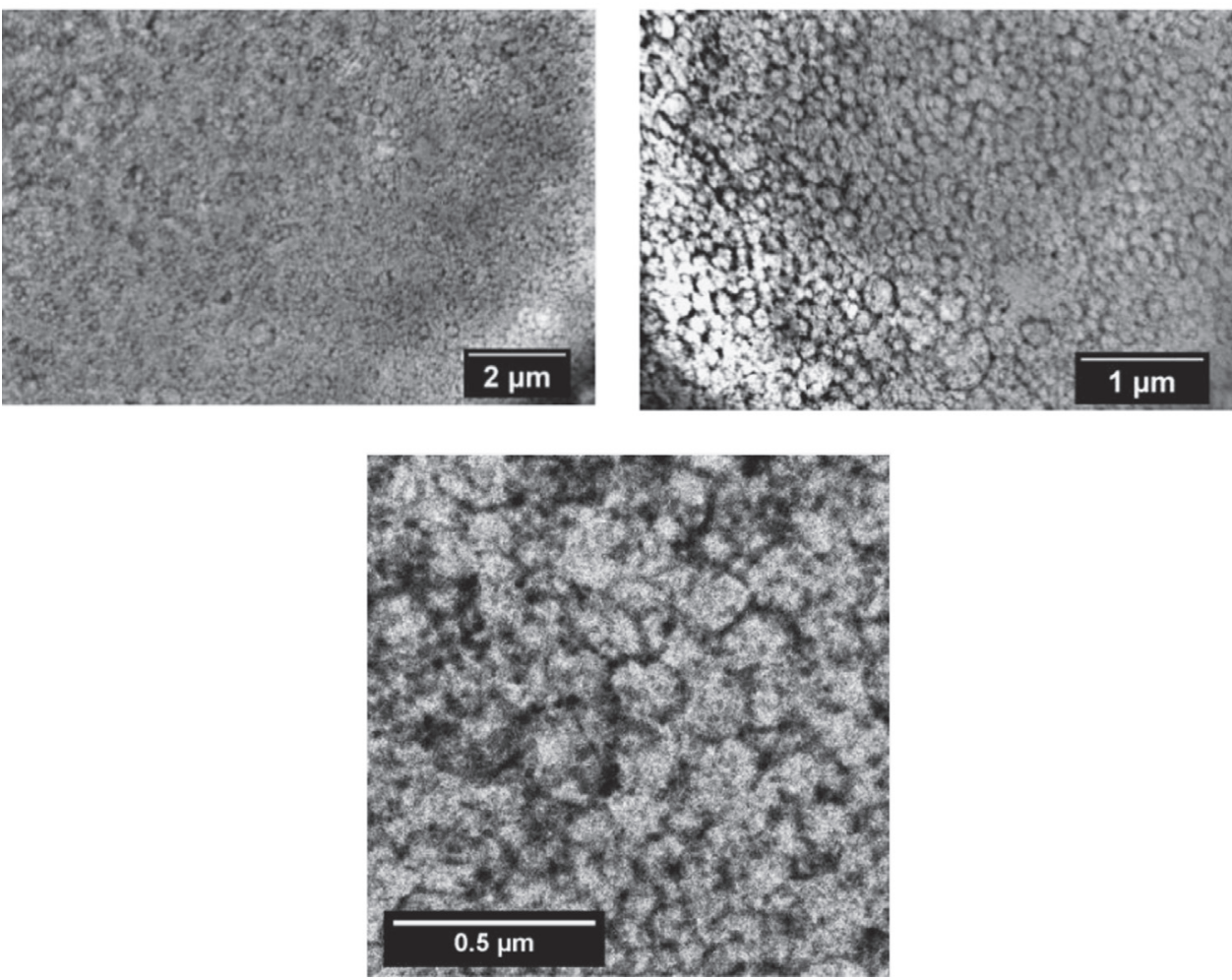

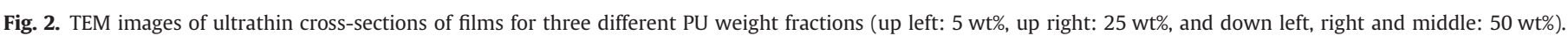

It is clear that the increase in the PU weight fraction modifies strongly the particle and film morphologies. This modification has consequences on the appearance of the film and on its mechanical and adhesive properties. The first point is that the dry film is typically crack-free and transparent when only $5 \mathrm{wt} \%$ PU is added, while for the highest PU weight fraction (50 wt\%), the final film is 
slightly cloudy. This slight opacity indicates some phase separation at a larger scale which induces changes in refractive index.

Mechanical properties of adhesive PU-acrylic hybrid films in uniaxial tension were analyzed in our previous study [15]. An increase of the elastic modulus and a decrease of the viscous character with increasing PU weight\% in the film were clearly observed. These trends were interpreted uniquely with molecular arguments, i.e. grafting of PU on the acrylic chains by double bonds and an increase in entanglement density due to the presence of the PU chains. Any change in the particles morphology was neglected whereas Figs. 1 and 2 show that it exists and should be considered. We present here a novel approach for these mechanical properties taking into account the adhesive film morphologies.

In Fig. 3, the large strain elastic properties in uniaxial tension of self-standing films obtained from the drying of PU/acrylic hybrid particles are presented. In addition to the classical normal stress vs. strain curve, the Mooney representation is added. The reduced stress $\sigma_{\mathrm{R}}$, also named Mooney stress, is defined as

$\sigma_{\mathrm{R}}=\frac{\sigma_{\mathrm{N}}}{\left(\lambda-\left(1 / \lambda^{2}\right)\right)}$

The reduced stress (which has the meaning of an effective strain dependent modulus) is typically represented vs. the inverse of strain $1 / \lambda$ and is independent of $\lambda$ for an elastomer following the classical rubber elasticity. For a typical PSA it is however strongly $\lambda$-dependent and typically decreases with $\lambda$ initially (a strain softening) and eventually increases at very large strain (a strain stiffening).

This representation highlights the onset of strain stiffening observed at large strain in this type of viscoelastic material [25]. This stiffening corresponds to the minimum observed on the curve at small $1 / \lambda$ and characterizes the finite extensibility of the film. Two important parameters can be extracted from these curves: the minimum value of reduced stress $C_{\text {hard }}$ and the value of stretch at that minimum, $\lambda_{\text {hard. }}$ The interpretation of the softening and stiffening can be molecular: relaxation of pendant chains and slippage of entanglement points for the softening, finite extensibility of the chains for the hardening [26]. In waterborne systems it can however also be due to the structure of the particle or of the film: percolating structure of shells for example [27-29].

This finite extensibility can be attributed to the maximum extension of the connected polymer chain structure. We also use $\lambda_{\text {hard }}$, the strain corresponding to the minimum reduced stress, which is the stiffness of the fully relaxed material. The strain values when samples break, $\lambda_{\text {break, }}$ were also noted and both values of $\lambda$ are reported in Table 2 for two different initial strain rates.

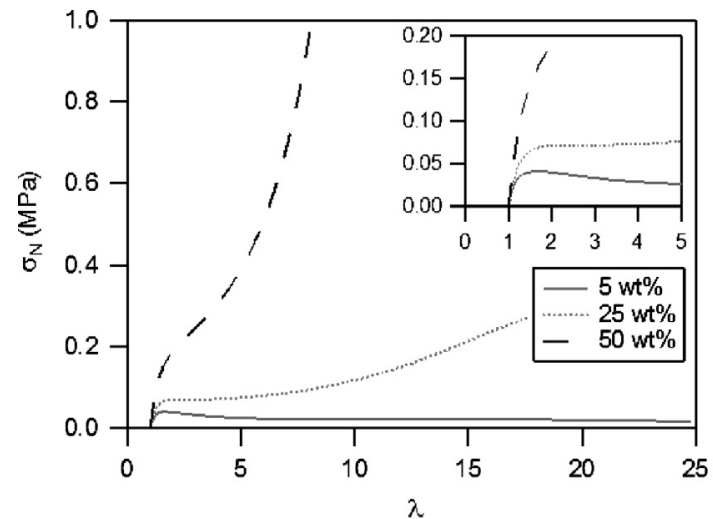

The shapes of the three curves (classic and Mooney graphs) characterize the transition from a very soft uncrosslinked film (for $5 \mathrm{wt} \%$ of PU) to a well crosslinked, mostly elastic sample (for $50 \mathrm{wt}$ $\%$ ). For $5 \mathrm{wt} \%$ of PU, no minimum in $\sigma_{\mathrm{R}}$ is observed at high $\lambda$ (low $1 / \lambda$, due to a lack of stiffening of the system. A direct consequence is that neither $\lambda_{\text {hard }}$ nor $C_{\text {hard }}$ can be reported. Instead, the sample flows, and failure occurs at very high $\lambda$. At the intermediate PU content a percolating network of chains is formed and the behavior resembles that of a standard PSA. However as the PU content is increased to $50 \mathrm{wt} \%$ the initial modulus and above all the value of $C_{\text {hard }}$ increase by a decade, while $\lambda_{\text {hard }}$ and $\lambda_{\text {break }}$ dramatically decrease. Such a decrease of the maximum deformation with the PU content has been observed by Peruzzo and coworkers [9] (for coating formulations), but the non-linearity observed for $50 \mathrm{wt} \%$ is a signature of the specific film morphology.

Tensile experiments were performed at two different nominal strain rates: $\mathrm{d} \varepsilon / \mathrm{d} t=0.1 \mathrm{~s}^{-1}$ and $\mathrm{d} \varepsilon / \mathrm{d} t=1 \mathrm{~s}^{-1}$. For each $\lambda$ the ratio $R(\lambda)$ between the stresses at the two different nominal strain rates was calculated. The objective was to separate the two components of the softening at intermediate strain: the reversible straindependent non-linear elasticity and the strain rate-dependent viscoelastic process. The representation of the ratio $R(\lambda)$ as a function of $\lambda$ (Fig. 4) is an empirical method which emphasizes the strain rate-dependent viscoelasticity [28]. A constant ratio $R(\lambda)$ indicates that the two processes are fully independent whereas any variation of this ratio with $\lambda$ reveals non-linear viscoelastic processes, i.e. the viscoelastic behavior depends on the strain. Moreover, the absolute value of $R(\lambda)$ is an indication of the level of viscoelasticity in the sample.

Three different behaviors are observed depending on the PU content. For $5 \mathrm{wt} \% \mathrm{PU}$, the ratio $R(\lambda)$ is very high and decreases slightly with $\lambda$. This is consistent with the results shown in Fig. 3 for a highly viscoelastic liquid. The film with intermediate level of PU becomes a viscoelastic solid and its $R(\lambda)$ is lower and relatively constant. Finally, the last sample (50 wt\% of PU) shows a $R(\lambda)$ close to 1 , indicating no large effect of the strain rate. This is consistent

Table 2

Maximum strain measured before failure for the three PU weight fractions.

\begin{tabular}{llrr}
\hline PU (wt\%) & 5 & 25 & \multicolumn{1}{c}{50} \\
\hline$E\left(\mathrm{MPa}\right.$, initial $\left.\mathrm{d} \varepsilon / \mathrm{d} t=1 \mathrm{~s}^{-1}\right)$ & 141.4 & 275.6 & 459.9 \\
$C_{\text {hard }}\left(\mathrm{kPa}\right.$, initial $\mathrm{d} \varepsilon / \mathrm{d} t=0.1 \mathrm{~s}^{-1}$ ) & $/$ & 6.8 & 66.9 \\
$C_{\text {hard }}\left(\mathrm{kPa}\right.$, initial $\left.\mathrm{d} \varepsilon / \mathrm{d} t=1 \mathrm{~s}^{-1}\right)$ & $/$ & 11.8 & 77.9 \\
$\lambda_{\text {hard }}\left(\right.$ initial $\left.\mathrm{d} \varepsilon / \mathrm{d} t=0.1 \mathrm{~s}^{-1}\right)$ & $/$ & 9.2 & 5.2 \\
$\lambda_{\text {hard }}\left(\right.$ initial $\left.\mathrm{d} \varepsilon / \mathrm{d} t=1 \mathrm{~s}^{-1}\right)$ & $/$ & 9.2 & 4.5 \\
$\lambda_{\text {break }}\left(\right.$ initial $\left.\mathrm{d} \varepsilon / \mathrm{d} t=0.1 \mathrm{~s}^{-1}\right)$ & 17.7 & 18.5 & 10.6 \\
$\lambda_{\text {break }}\left(\right.$ initial $\left.\mathrm{d} \varepsilon / \mathrm{d} t=1 \mathrm{~s}^{-1}\right)$ & 25.1 & 25.9 & 8.2 \\
\hline
\end{tabular}

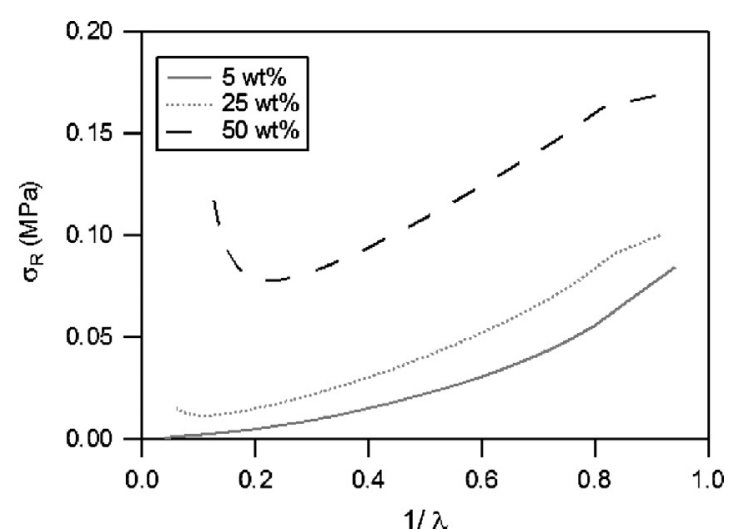

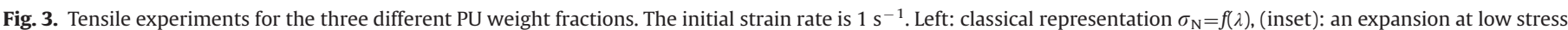
and strain. Right: Mooney representation $\sigma_{\mathrm{R}}=f(1 / \lambda)$. 


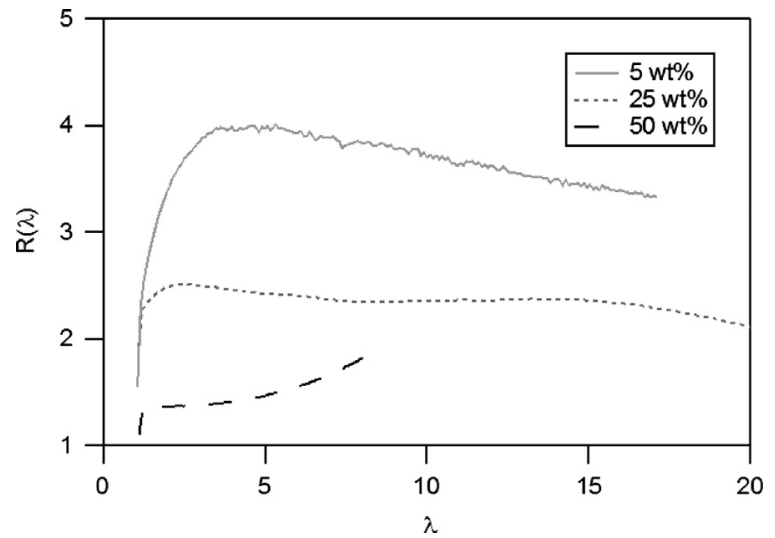

Fig. 4. Evolution of the strain rate ratio $R(\lambda)$ with the strain $\lambda$ for the three different PU weight fractions.

with the hypothesis of a predominantly elastic sample. In addition the shape of this last curve is particularly interesting. Indeed, $R(\lambda)$ increases from 1.4 when the softening starts to 1.8 at the end of the curve. The sample becomes more dissipative at higher strains which is consistent with a failure of the interfaces between particles.

Results from Figs. 3 and 4 indicate that the change in the morphology between $25 \mathrm{wt} \%$ and $50 \mathrm{wt} \%$ of PU strongly modifies the large strain behavior ( $C_{\text {hard }}, \lambda_{\text {hard }}$, and $\left.\lambda_{\text {break }}\right)$. Such a brutal change could not correspond to a simple increase of the density of crosslinking points in the polymer network architecture due to an increase of the PU incorporation in the network, but is obviously correlated with the specific morphology observed in the film. For $50 \mathrm{wt} \%$, the PU-acrylic partial phase separation with a PU-rich region in the shell results in a stiffer and percolating network of shells and limits the diffusion of the acrylic chains between particles during the latex drying process. Two reasons can explain this reduced interdiffusion. First, acrylics and PU have a low compatibility. Second, PU rich domains are made predominantly of PPG segments which are shorter and probably more densely crosslinked compared to the acrylic chains. These short densely crosslinked chains have less facility to diffuse between particles. The consequence of a low level of interdiffusion is that interfaces between particles are weak and can withstand lower stresses than the particle itself. At a macroscopic scale, this translates in an abrupt modification of the mechanical properties.

It is clear that when changing the PU content at a fixed grafting degree, the quantity of functional monomer HEMA also changes because the ratio $\mathrm{NCO} / \mathrm{OH}$ is kept constant. In a previous paper [15], we showed that an increase in PU content at fixed grafting degree (i.e. increasing HEMA content) increased the crosslink density of the polymer network. This denser network should also limit the interdiffusion of chains across particles and is consistent with the observed trend.

Very interesting results on the modeling of the polymer architecture inside the particle of complex PU/acrylic hybrids systems have also been reported by Lopez et al. [12,30,31]. These studies assumed however that the particles were homogeneous. The present work provides solid evidence that acquiring information and knowledge on particles and films morphology is a necessary complement to molecular arguments for a correct interpretation of the macroscopic properties.

\section{Conclusion}

We have examined in this paper the relationship between morphology and macroscopic properties of adhesive films with complex polymer architecture and particle morphology. Soft tacky PU/acrylic hybrid particles and films obtained upon drying such particles have been successfully imaged by TEM. Whereas the films were considered to be homogeneous at the scale of the polymer network in previous work, the TEM images clearly indicate a significant degree of phase separation at high PU contents. Thus, while at $5 \mathrm{wt} \%$ and $25 \mathrm{wt} \% \mathrm{PU}$, the particles are predominantly homogeneous, at 50 wt\% PU a core-shell morphology is observed, with PU-rich domains preferentially located in the outer shell. This suggests that the incorporation of PU in the particles through chemical grafting to the acrylic chains is probably not a sufficient condition to have homogeneous hybrid particles when the PU weight fraction is higher than $25 \mathrm{wt} \%$.

The core-shell morphology persists in the adhesive films, resulting in a percolating network of stiffer PU-rich shells. The reduced miscibility between the two phases limits particle interpenetration and hence the interfacial strength, resulting in dramatic changes in the film properties: the maximum extension is reduced while the stiffness increases markedly, making the films unsuitable for PSA applications. These abrupt changes in properties can only be explained in terms of the aforementioned morphological changes.

\section{References}

[1] Hirose M, Kadowaki F, Zhou JH. The structure and properties of core-shell type acrylic-polyurethane hybrid aqueous emulsions. Prog Org Coat 1997;31:157-69.

[2] Kukanja D, Golob J, Zupancic-Vlant A, Kranjc M. The structure and properties of acrylic-polyurethane hybrid emulsions and comparison with physical blends. J Appl Polym Sci 2000;78:67-80.

[3] Li M, Daniel ES, Dimonie V, Sudol ED, El-Aasser M. Preparation of polyurethane/acrylic hybrid nanoparticles via a miniemulsion polymerization process. Macromolecules 2005;38:4183-92.

[4] Hegedus CR, Kloiber KA. Aqueous acrylic-polyurethane hybrid dispersions and their use in industrial coatings. J Coat Technol 1996;68:39.

[5] Gooch JW, Dong H, Schork FJ. Waterborne oil-modified polyurethane coatings via hybrid miniemulsion polymerization. J Appl Polym Sci 2000;76:105-14.

[6] Wang C, Chu F, Graillat C, Guyot A. Hybrid acrylic-polyurethane latexes by miniemulsion polymerization. Polym React Eng 2003;11:541-62.

[7] Wang C, Chu F, Guyot A. Mechanical properties of films from hybrid acrylic/ polyurethane polymer colloids. J Dispers Sci Technol 2006;27:325-30.

[8] Wang CP, Chu FX, Jin LW, Lin MT, Xu YZ, Guyot A. Polyurethane-acrylate hybrid latexes from miniemulsion polymerization: effect of endgroups on structure and properties. Polym Adv Technol 2009;20:319-26.

[9] Peruzzo PJ, Anbinder PS, Pardini OR, Costa CA, Leite CA, Galembeck F, et al. Polyurethane/acrylate hybrids: effects of the acrylic content and thermal treatment on the polymer properties. J Appl Polym Sci 2010;116:2694-705.

[10] Tian C, Zhou Q, Cao L, Su Z, Chen X. Effect of polyurethane molecular weight on the properties of polyurethane-poly(butyl methacrylate) hybrid latex prepared by miniemulsion polymerization. J Appl Polym Sci 2012;124: 5229-5235.

[11] Guo Y, Li S, Wang G, Ma W, Huang Z. Waterborne polyurethane/poly(n-butyl acrylate-styrene) hybrid emulsions: particle formation, film properties, and application. Prog Org Coat 2012;74:248-56.

[12] Lopez A, Degrandi E, Canetta E, Keddie JL, Creton C, Asua JM. Simultaneous free radical and addition miniemulsion polymerization: effect of the diol on the microstructure of polyurethane-acrylic pressure-sensitive adhesives. Polymer 2011;52:3021-30.

[13] Agirre A, Nase J, Degrandi E, Creton C, Asua JM. Improving adhesion of acrylic waterborne PSAs to low surface energy materials: introduction of stearyl acrylate. J Polym Sci Part A: Polym Chem 2010;48:5030-9.

[14] Agirre A, Nase J, Degrandi E, Creton C, Asua JM. Miniemulsion polymerization of 2-ethylhexyl acrylate. Polymer architecture control and adhesion properties. Macromolecules 2010;43:8924-32.

[15] Degrandi-Contraires E, Udagama R, Bourgeat-Lami E, McKenna T, Ouzineb K, Creton C. Mechanical properties of adhesive films obtained from PU-acrylic hybrid particles. Macromolecules 2011;44:2643-52.

[16] Degrandi-Contraires E, Lopez A, Reyes Y, Asua JM, Creton C. High-shearstrength waterborne polyurethane/acrylic soft adhesives. Macromol Mater Eng 2012;298:612-23.

[17] Udagama R, Degrandi-Contraires E, Creton C, Graillat C, McKenna TFL, Bourgeat-Lami E. Synthesis of acrylic-polyurethane hybrid latexes by miniemulsion polymerization and their pressure-sensitive adhesive applications. Macromolecules 2011;44:2632-42.

[18] Lopez A, Degrandi-Contraires E, Canetta E, Creton C, Keddie JL, Asua JM. Waterborne polyurethane-acrylic nanocomposite nanoparticles by 
miniemulsion polymerization: applications as pressure-sensitive adhesives. Langmuir 2011;27:3878-88.

[19] Creton C. Pressure-sensitive-adhesives: an introductory course. MRS Bull 2003;28:434-9.

[20] Hirose M, Zhou JH, Nagai K. The structure and properties of acrylic-polyurethane hybrid emulsions. Prog Org Coat 2000;38:27-34.

[21] Chiu H-T, Yang H-M, Liu C-S, Hsu H-Y. Synthesis, stability and properties of polyurethane/acrylic hybrids using m-TMXDI-based anionic poly(urethaneurea) dispersion. Polym-Plast Technol Eng 2012;51:945-53.

[22] Chai SL, Jin MM, Tan HM. Comparative study between core-shell and interpenetrating network structure polyurethane/polyacrylate composite emulsion. Eur Polym J 2008;44:3306-13.

[23] Li C-Y, Chiu W-Y, Don T-M. Morphology of PU/PMMA hybrid particles from miniemulsion polymerization: thermodynamic consideration. J Polym Sci Part A: Polym Chem 2007;45:3359-69.

[24] Sebenik U, Krajnc M. Properties of acrylic-polyurethane hybrid emulsiosn synthesized by the semibatch emulsion copolymerization of acrylates using different polyurethane particles. J Polym Sci Part A: Polym Chem 2005;43:4050-69.
[25] Deplace F, Carelli C, Mariot S, Retsos H, Chateauminois A, Ouzineb K, et al Fine tuning the adhesive properties of a soft waterborne adhesive from rheological measurements. J Adhes 2009;85:18-54.

[26] Roos A, Creton C. Effect of the presence of diblock copolymer on the nonlinear elastic and viscoelastic properties of elastomeric triblock copolymers. Macromolecules 2005;38:7807-18.

[27] Deplace F, Rabjohns MA, Yamaguchi T, Foster AB, Carelli C, Lei C-H, et al Deformation and adhesion of a periodic soft-soft nanocomposite designed with structured polymer colloid particles. Soft Matter 2009;5:1440.

[28] Bellamine A, Degrandi E, Gerst M, Stark R, Beyers C, Creton C. Design of nanostructured waterborne adhesives with improved shear resistance. Macromol Mater Eng 2011;296:31-41.

[29] Rharbi Y, Boué F, Joanicot M, Cabane B. Deformation of cellular polymeric films. Macromolecules 1996;29:4346-59.

[30] Lopez A, Reyes Y, Degrandi-Contraires E, Canetta E, Creton C, Keddie JL, et al. Simultaneous free-radical and addition miniemulsion polymerization: effect of the chain transfer agent on the microstructure of polyurethane-acrylic pressure-sensitive adhesives. Macromol Mater Eng 2012;298:53-66.

[31] Reyes Y, Lopez A, Asua JM. Modeling the microstructure of acrylic-polyurethane hybrid polymers synthesized by miniemulsion polymerization. Macromol React Eng 2011;5:352-60. 